\title{
What is Karyomapping and where does it fit in the world of preimplantation genetic diagnosis (PGD)?
}

\author{
Authors: \\ Darren K. Griffin \\ Rebecca L. Gould \\ School of Biological Sciences, \\ University of Kent, \\ Canterbury CT2 7NJ. UK \\ The Bridge Centre, $1 \mathrm{St}$ \\ Thomas St, London, SE1 \\ 9RY. UK
}

\begin{abstract}
The first application of preimplantation genetic diagnosis (PGD) recently celebrated its $25^{\text {th }}$ birthday. Aside from the very early days when chromosomal diagnoses were used (by sexing) for the selective implantation of embryos unaffected by sex linked disorders, the paths of chromosomal and monogenic PGD have diverged. For monogenic disorders, progress has been impeded by the need to tailor each diagnosis to the mutation in question. For chromosomal diagnoses, fluorescent in situ hybridization (FISH) technology was replaced by array comparative genomic hybridization $(\mathrm{aCGH})$, and then next generation sequencing (NGS). Karyomapping is a novel approach that allows the detection of the inheritance of (grand) parental haploblocks through the identification of inherited chromosomal segments. It involves genome-wide single nucleotide polymorphism (SNP) analysis of parental DNA, a reference from a related individual of known disease status (typically an affected child) and amplified DNA form biopsied cells of the (usually blastocyst) embryos in question. Identification of informative loci for each of four parental haplotypes is followed by direct comparison to the reference, ultimately creating a Karyomap. The Karyomapping programme (Illumina) displays homologous chromosomes, points of crossing over and the haplotype of each of the embryos. It also detects meiotic trisomy, monosomy, triploidy and uniparental heterodisomy (some of which NGS and aCGH will not). Inherent in the design is the analysis of "key SNPs" (heterozygous informative calls) thereby avoiding the risk of misdiagnoses caused by the phenomenon of allele drop out (ADO). Karyomapping is currently in use for the detection of monogenic disorders and around 1000 clinics offer it worldwide making use of about 20 diagnostic laboratories. At the time of writing, over two and a half thousand clinical cases have been performed. Because of the limited detection of some post-zygotic errors such as post-zygotic trisomy which can also lead to mosaicism, Karyomapping has not yet been fully applied clinically for aneuploidy screening. The diagnostic potential of the technique will be fully recognised with the application of this technology on clinical cases.
\end{abstract}


What is Karyomapping and where does it fit in the world of preimplantation genetic diagnosis (PGD)?

\section{A brief history of PGD}

Preimplantation genetic diagnosis (PGD) involves the genetic testing of oocytes or IVF embryos facilitating selective transfer of apparently genetically normal embryos (Handyside, 2011). PGD collectively refers to the diagnosis of monogenic disorders, human leukocyte antigen (HLA) typing, detection of unbalanced chromosome rearrangements ahead of implantation to reduce the risk of babies born with congenital abnormalities or implantation failure and/or pregnancy loss (Braude et al., 2002; Frumkin et al., 2008; Harton et al., 2011b). The first reported case of PGD in non-human species was sex selection of rabbits performed by Bob Edwards and Richard Gardner (Gardner and Edwards, 1968). In 1990, the first clinical cases in humans were performed for two couples at risk of transmitting $\mathrm{X}$-linkedAdrenoleukodystrophy (X-ALD) and Xlinked mental retardation (Handyside et al., 1990) with the use of sex-chromosome specific primers. Later the first PGD for an autosomal recessive monogenic disorder (Cystic Fibrosis, $\triangle$ F508 mutation of the CFTR gene) was announced (in 1992) (Handyside et al., 1992). As previously alluded to, PGD has come to refer not only the detection of specific monogenic traits but also the elimination of chromosomally unbalanced products from rearrangement carriers and the selection of human leukocyte antigen (HLA) identical embryos which might serve as potential 'saviour siblings' for affected individuals in need of bone-marrow transfer (Kahraman et al., 2014). In contrast, preimplantation genetic screening (PGS), sometimes referred to as PGD-A, specifically pertains to aneuploidy assessment in embryos e.g. for referral categories such as advanced maternal age, recurrent implantation failure or recurrent miscarriage (Delhanty et al., 1993; Geraedts and De Wert, 2009; Geraedts, 2010; Harper and SenGupta, 2012b; Traeger-Synodinos and Staessen, 2014). PGD can be applied to detect any single gene mutation, but the "headline" diseases tend to be cystic fibrosis, beta-thalassemia, myotonic dystrophy, Huntington's disease and fragile X (Harper and SenGupta, 2012b; Spits and Sermon, 2009). However, the 'holy grail' of PGD is a single universal test that will detect any monogenic and all chromosomal disorders simultaneously (Handyside, 2015; Thornhill et al., 2015).

Historically, it was the development of polymerase chain reaction (PCR) to be sensitive enough to work on single cells that heralded techniques for mutation detection in this setting (Ben-Nagi et al., 2016; Handyside et al., 2004; Thornhill and Snow, 2002). The inherent problems associated with such diagnostic tests however lie in their sensitivity and in the issue of contamination. In terms of sensitivity, the tiny amount of DNA (5-10pg) present in a single cell that needs to be analysed raises problems of amplification failure and allele drop out (ADO) (i.e. allele specific amplification that could lead to a heterozygote being diagnosed as a homozygote). Regarding contamination, all precautions must be taken to ensure that no amount of contaminating DNA enters the reaction tube (Thornhill and Snow, 2002). This has been a problem from even the earliest days of PGD (Handyside et al., 1990) even when amplifying repetitive $Y$ specific sequences (e.g. (Chong et al., 1993; Hashiba et al., 1999; Levinson et al., 1995; Nakahori et al., 1991). Nested PCR (a second round of singleplex PCR using the first multiplex as a template) increased specificity and thus the ability to detect diseases such as cystic fibrosis and Alpha-1 Antitrypsin (A1AT) deficiency (Handyside et al., 1992). Countless other mutations followed (e.g. (Dahdouh et al., 2015; Hussey et al., 1999; Liu et al., 1995; Ray et al., 2001). 
What is Karyomapping and where does it fit in the world of preimplantation genetic diagnosis (PGD)?

Human genome sequencing data (International Human Genome Sequencing Consortium, 2004; Lander et al., 2001; Sachidanandam et al., 2001; Venter et al., 2001) revealed polymorphic markers that can be used in a multiplex PCR strategy (Thornhill et al., 2015). In particular, analysis of short tandem repeat (STR) markers (principally tetranucleotide repeats) facilitated analysis not only of the mutation in question but of linked markers around the gene. Such strategies minimised the problems caused by ADO and contamination as each individual analysis confirms the other (Harton et al., 2011a; Thornhill et al., 2015). The technology was taken a step further with the adoption of whole genomic amplification e.g. multiple displacement amplification (MDA) (Handyside et al., 2004; Hellani et al., 2008), facilitating multiplex PCRs and chromosome copy number detection (Dahdouh et al., 2015) see later. PCR with fluorescently labelled primers also facilitated more accurate detection and multiplexing (Ao et al., 1998; De Rycke, 2010; Spits and Sermon, 2009). Using this approach over a 10-year period (1997-2007) they reported that the misdiagnosis rate was $10 / 3727 \quad(0.27 \%)$ (Ben-Nagi et al., 2016; Harper et al., 2012a). This also allowed for analysis of so called saviour siblings (Handyside and $\mathrm{Xu}, 2012$; Renwick et al., 2010) and ultimately became the established approach for PGD (Fiorentino et al., 2004; Handyside, 2015). The main issue with this approach is that it needs to be tailored to the patients in question, leading to an inevitable (and often stressful) waiting period where primers are designed and tested for couples. Furthermore, it was limited in its ability to detect chromosome abnormalities (Ata et al., 2012; Konstantinidis et al., 2015; Munné, 2006; Munné et al., 1996; Thornhill et al., 2015).

Following the ultimately unreliable PCR sexing strategies attempts to sex preimplantation embryos (Handyside et al., 1990), attention turned to fluorescence insitu hybridisation (FISH) using sex chromosome specific probes (Griffin et al., 1991). Clinical application followed (Griffin et al., 1994; Griffin et al., 1992; Griffin et al., 1993) from 1992-1994, 27 treatment cycles ensued, leading to 9 in and 5 female live births (Delhanty et al., 1993; Griffin et al., 1994; Griffin et al., 1993). FISH was then applied to translocation detection and aneuploidy screening (Munné et al., 1996; Munné and Cohen, 1993; 1998; Munné et al., 1994) and became the most common form of PGD. Referral categories were advanced maternal age (AMA), recurrent miscarriage (RM), recurrent implantation failure (RIF) and severe male factor infertility. The approach made use of fluorescent probes chiefly for chromosomes 13, 16, 18, 21, 22 (X and Y) (Munné et al., 1996; Munné and Cohen, 1993; 1998; Munné et al., 1994) but was the subject of controversy in the field (Summers and Foland, 2009), with randomized controlled trials indicating no clear benefit for using it. It is now a matter of historical record that one study (Mastenbroek et al., 2007) provided evidence that there was a detrimental effect of PGS, with others demonstrating no benefit (Blockeel et al., 2008; Debrock et al., 2010; Hardarson et al., 2008; Jansen et al., 2008; Mersereau et al., 2008; Meyer et al., 2009; Schoolcraft et al., 2009; Staessen et al., 2008), and 5-7 colour FISH strategies were largely discontinued. Even though 24 chromosome FISH was successfully applied to human embryos (Ioannou et al., 2012; Ioannou et al., 2011) there were technical problems with overlapping signals, failed probes, etc. condemning FISH to being a research tool only (albeit a useful one) for looking at phenomena such as nuclear organization and mosaicism. FISH still persists in some PGD clinics however array comparative genomic hybridization (aCGH) and next generation sequencing (NGS) have largely replaced it. 
What is Karyomapping and where does it fit in the world of preimplantation genetic diagnosis (PGD)?

Comparative genomic hybridisation $(\mathrm{CGH})$ took the principles of FISH one step further, by using differentially labelled test and control (normal) DNA, that are competitively hybridized to metaphase chromosomes (Theisen, 2008). Unlike FISH, $\mathrm{CGH}$ allowed for the more rapid assessment of chromosome copy number in the entire genome (Forozan et al., 1997; Spelcher et al., 1993) but was limited to a resolution of approximately $5-10 \mathrm{Mb}$ in most clinical applications (Kirchhoff et al., 1998; Lichter et al., 2000). Due to its limitations, this technology fell out of favour for clinical diagnosis, therefore other techniques were developed, including aCGH and NGS as mentioned previously.

Both aCGH and NGS require whole genome amplification strategies such as multiple displacement amplification (MDA) or degenerate oligonucleotide primed-PCR (DOP-PCR). Array $\mathrm{CGH}$ involves fluorescent labeling of a test DNA sample and a reference (normal) in two different fluorescent colours (De Ravel et al., 2007). Simultaneous hybridization to a tiling path microarray precedes per-chromosome colour ratio analysis - deviations from 1:1 are indicative of aneuploidy, deletions and duplications (Fishel et al., 2010; Le Caignec et al., 2006; Traversa et al., 2011; Vanneste et al., 2009). At a similar time, Treff and colleagues developed and validated real-time quantitative PCR (RT-qPCR) (Scott et al., 2013b; Treff and Scott, 2013b; Treff et al., 2012). In RT-qPCR samples undergo a preamplification step, followed by a high-order multiplex PCR reaction to amplify two regions on each arm of all the chromosomes. Rapid quantification of each product using RT-qPCR then allows for the evaluation of copy number over the whole genome (Dahdouh et al., 2015). Using this technique, PCR is performed directly on the sample, without a whole genome amplification (WGA) step required in technology such as aCGH and SNP microarrays (see below).
However, this means that RT-qPCR can only be used on trophectoderm samples, and so sufficient blastocyst embryos need to be available (Dahdouh et al., 2015; Treff et al., 2012).

Around the time of the aCGH renaissance, biopsy strategies switched from cleavage stage to blastocyst stage, and aCGH has recently largely been replaced by NGS due to the availability of benchtop sequencing technology. To detect aneuploidy by this approach fragmentation of the whole genome amplified DNA into 100-200 base pairs pieces is essential. Sequencing using fluorescent tags ensues to indicate the relevant sample, facilitating low cost, high throughput analysis (Fiorentino et al., 2014). Sequence data is compared with a reference genome and then the process involves simple "binning" and counting of sequences to generate copy number information (Fiorentino et al., 2014). In other words, the number of sequences counted from a specific chromosome is proportional to the chromosome copy number, leading to detection of monosomy, trisomy or segmental alterations (Handyside, 2013; Handyside and Wells, 2013). NGS has a greater dynamic range than aCGH and represents the current state of the art for aneuploidy detection for PGD.

\section{Single nucleotide polymorphism arrays (SNP chips)}

Specialist microarrays detect thousands of SNPs across the genome in a range of cell types including human embryos (Handyside, 2015). Initially developed for genome wide association studies (GWAS), SNP chips represent biallelic loci where each allele is of roughly a similar frequency (Habela and Hamosh, 2013; LaFramboise, 2009). Probing SNP chips leads to each allele being differentially fluorescently labelled thereby detecting homozygotes and heterozygotes and a binary readout at each 
What is Karyomapping and where does it fit in the world of preimplantation genetic diagnosis (PGD)?

locus e.g. AA, AB, BB. SNPS are located mostly in non-coding regions (around 40 million are validated in the human genome) with SNP chips typically containing 660,000 to 2 million loci spread roughly evenly along the length of all human chromosomes (Dahdouh et al., 2015).

As SNPs are biallelic in nature, they are theoretically less informative than STR markers (Handyside, 2015). However, given the information of parental genotype and a relative of known disease status, the four distinct sets of markers can be identified along the length of each parental chromosome for each tested SNPs (Handyside, 2015). Such an approach has been used for linkage analysis for monogenic disease detection (Rabinowitz et al., 2011) and detection of chromosome abnormalities (Treff et al., 2011) (Dahdouh et al., 2015); the Karyomapping technique combines these two strategies.

\section{Karyomapping}

Karyomapping (Handyside et al., 2010) determines the inheritance from the parental (or grandparental) chromosomes through the recapitulation of haploblocks or inherited chromosomal segments). The approach involves genome-wide SNP analysis of parental DNA, amplified embryo DNA and an appropriate 'reference' such as a close relative (typically an older, affected child is used).

The initial stage is the identification of 'informative' loci for each of the parental haplotypes at which where parent is homozygous and another heterozygous (Natesan et al., 2014a). This is analogous to a "backcross" that we learn about in genetics 101. These informative loci are then compared to the reference DNA in order to establish phase (assign a reference "affected" haplotype). After this the genotype of each embryo is compared to the reference to establish similarity or difference at each informative locus. The result is a karyomap (figure 1) that displays homologous chromosomes as well as points of meiotic crossing over.

Direct comparison of the haploblock at the chromosomal locus of interest in the reference with those of each of the embryos in question (figure 1) allows the diagnosis of the presence or absence of a disease causing allele (Handyside, 2015). This is achieved using the principles of genetic linkage established by Morgan and Sturtevant in the 1930s and does not involve direct mutation testing. One of the important advantages of Karyomapping is that it can also be applied for the diagnosis of meiotic trisomy (figure 1 right), monosomy, triploidy, parthenogenetic activation and uniparental heterodisomy; as well as patterns of genomic duplication seen in, for example, hydatidiform moles (Handyside et al., 2010; Natesan et al., 2014a; Natesan et al., 2014b). When analysing the Karyomapping programme output, monosomies and segmental deletions are visualised as absence of haploblocks from one parent. Meiotic (but not postzygotic) trisomies are visualised as both haplotypes on a section of the chromosome, reduced to homozygosity after a crossing over event. Furthermore, meiosis I and meiosis II errors can potentially be distinguished depending on whether the "double haplotype" pattern involves the centromere of the chromosome (Handyside et al., 2010). Moreover, Karyomapping only involves the detection of heterozygous SNP calls. These are termed "key SNPs" and, by gathering the information from thousands of key SNPs, the misdiagnosis risks associated with ADO are eliminated. 
What is Karyomapping and where does it fit in the world of preimplantation genetic diagnosis (PGD)?

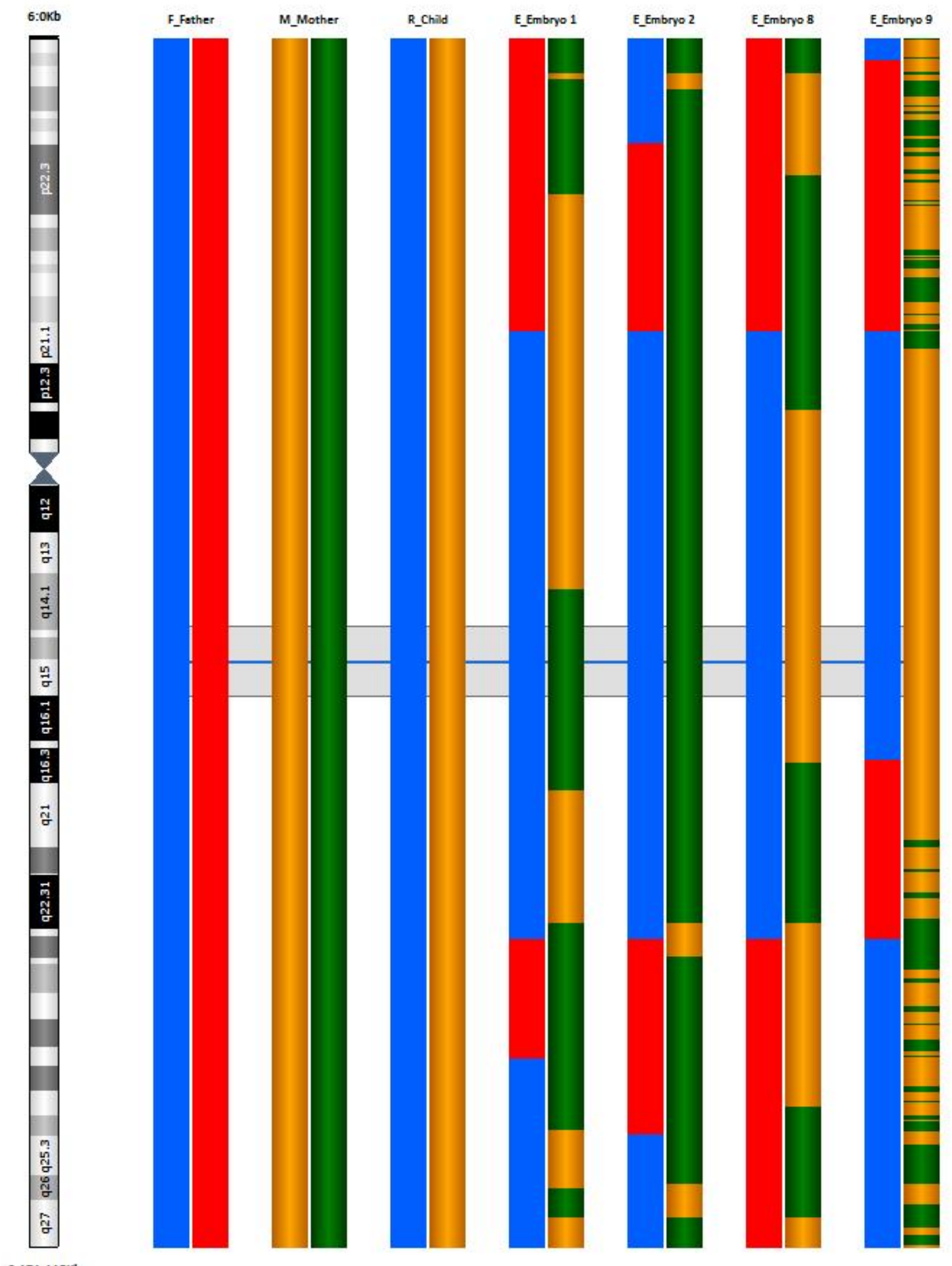

Figure 1. Karyomapping output of the RARS2 gene on chromosome 6q15 (linked to pontocerebellar hypoplasia). From left to right, the red and blue bars (far left) are the paternal genotype, the yellow and green bars the maternal genotype. The blue and yellow bars are the reference (affected sibling), the remainder are embryonic genotypes showing (grand)parental inheritance of haploblocks. In embryo 9 (far right) the rapid alternating yellow and green bars represent a meiotic maternal trisomy (probably meiosis II as the pattern is not present around the centromere). 
What is Karyomapping and where does it fit in the world of preimplantation genetic diagnosis (PGD)?

Karyomapping therefore facilitates the simultaneous detection of monogenic and chromosomal disorders in a single test requiring only minimal patient work up (Ben-Nagi et al., 2016; Dahdouh et al., 2015; Handyside et al., 2010). When applied clinically, although Karyomapping can be used on any SNP chip with reasonable genome coverage (or in principle on NGS data) it uses exclusively a dedicated Illumina SNP chip with $\sim 300,000$ SNPs and a bespoke software suite for visualization. Karyomapping was validated using a blinded retrospective analysis by comparison with an STR marker and mutation detection approach (see above). The original studies in this regard were two case reports where Karyomapping was run in parallel with conventional analysis for Smith Lemli Opitz Syndrome and Marfan Syndrome respectively (Natesan et al., 2014b; Thornhill et al., 2015). The first of these revealed full concordance with the clinical analysis of the samples by STR analysis and for aneuploidy testing (Natesan et al., 2014a; Natesan et al., 2014b). The second (Thornhill et al., 2015) established that both approaches diagnosed disease status with high efficiency and accuracy but that Karyomapping was a considerably more time-efficient process given that the prePGD patient work-up was much shorter (Thornhill et al., 2015). Both led to unaffected, chromosomally normal live births. A further study used Karyomapping for detection of TSC2 (tuberous sclerosis) mutations (Giménez et al., 2015). In this case Karyomapping was able to detect the mutation whereas conventional analysis was not and led to the birth of an unaffected child. (Giménez et al., 2015). Analysis of subsequent multiple cases compared Karyomapping with direct mutation analysis in 218 embryos from 44 IVF cycles. The authors established that Karyomapping produces the same result as mutation analysis in $213 / 218(97.7 \%)$ cases and that

Copyright 2017 KEI Journals. All Rights Reserved non-concordance was a result of consanguinity (Natesan et al., 2014b).

The potentially ubiquitous "platform independent" nature of Karyomapping infers a certain degree of "future proofing," particularly as the output is binary i.e. a series of $\mathrm{AA}, \mathrm{AB}$ and $\mathrm{BB}$ biallelic genotypes (Giménez et al., 2015). It is noteworthy however, that even when we consider continually improving whole genome amplification protocols, gaps are bound to appear for technical reasons associated with the amplification from such a small template. Karyomapping has the potential to combat this problem by refining the algorithms to predict the likely genotype by further linkage analysis (Giménez et al., 2015). In the future, there is a possibility of combining Karyomapping with allele intensity measurements for the detection of non-meiotic trisomies and duplications (Rabinowitz et al., 2012; Zamani Esteki et al., 2015a) thereby distinguishing meiosis I, meiosis II and post-zygotic errors (Giménez et al., 2015). This is clinically significant because mosaic trisomies of meiotic origin usually lead to clinical problems; however, those of post-zygotic origin can, in certain circumstances, lead to unaffected live births.

Whether we should use PGS at all still remains the topic of controversial debate; however, there is increasing evidence of its efficacy in improving IVF success and reducing the risk of miscarriage (Forman et al., 2013a; Forman et al., 2013b; Giménez et al., 2015; Scott et al., 2013b; Yang et al., 2012). From a clinical point of view, the additional information about the origin of the chromosomal error facilitated by Karyomapping (but not other PGD strategies) has the potential to aid couples to establish future treatment regimens; donor gametes may be an option (Thornhill et al., 2015).

Despite its many advantages, Karyomapping is limited in a number of 
What is Karyomapping and where does it fit in the world of preimplantation genetic diagnosis (PGD)?

areas. The inherent requirement of DNA from a family member with known disease status (e.g. affected sibling) particularly in dominant disorders that may shorten life span thereby (leading to such an individual not being available) is one example. Another is, if a meiotic cross over in either parent, reference individual or embryo juxtaposes the locus, this may lead to difficulties in data interpretation and thus an inconclusive diagnosis. Karyomapping will not detect de novo mutations other than those that are inherited by Mendelian pattern (Rechitsky et al., 2011). A final problem (hitherto already mentioned) is the fact that Karyomapping cannot easily detect post-zygotic trisomies unless combined with quantitative approaches such as NGS, aCGH or allele intensity measurement.

Although patient work up for Karyomapping is acknowledged to be shorter than that of other methods for mutation detection, disorders that are not already established by PGD technologies will need STR marker tests before Karyomapping can be used. In these cases, the work up time for Karyomapping is the same as other technologies. It is important to note that there are cost implications regarding the implementation of Karyomapping. If the lab follows the published Illumina protocol, Karyomapping requires 4 products: SureMDA ${ }^{\mathrm{TM}}$, DNA Analysis Kit, the iScan ${ }^{\circledR}$ System or NextSeq ${ }^{\circledR} 550$ System, and BlueFuse ${ }^{\circledR}$ Multi analysis software (Illumina 2015). The scanning system required to read the BeadChips is different for those required for NGS (VeriSeq ${ }^{\circledR}$ ), and Karyomapping requires MDA to amplify the DNA instead of PCR-based methods commonly used in aCGH and NGS. Due to these requirements, there is also a need for dedicated workrooms for each stage of sample preparation that adds to the logistical costs of running a Karyomapping assay.

\section{Alternatives to Karyomapping}

As previously mentioned, dectection of both monogenic disorders and chromosomal abnormalities simultaneously is of paramount importance, therefore different methods have been developed. One suggestion is the combination of classical PGD techniques, such as STR+direct mutation analysis combined with aneuploidy testing through the use of aCGH (Rechitsky et al., 2015). This method was shown to increase implantation rates and significantly reduce miscarriage rates, however it requires different experimental setup leading potentially to increased time and cost within the diagnostic laboratory (Rechitsky et al., 2015).

Treff et al. developed the use of RTqPCR for the simultaneous detection of monogenic disorders and aneuploidy in IVF derived human embryos. This technique is based around a targeted NGS strategy and a multiplex PCR reaction that had targeted the required mutation site and chromosomespecific target sequences (Treff et al., 2013a). Interestingly, with this technique the necessary read depth for accurate sequencing of the mutation site is reduced per chromosome copy number, which allows for a reduction in per sample cost as well as the time required to run the test (Dahdouh et al., 2015). Zimmerman and colleagues determined that this strategy was more reliable than other techniques (Zimmerman et al., 2016) with 303/304 (99.7\%) embryos getting a definitive diagnosis and 1/304 $(0.3 \%)$ recorded as inconclusive due to a recombination event. This study also demonstrated an $82 \%$ (27/33) pregnancy rate (Zimmerman et al., 2016).

Another interesting method is the use of NGS technology with linkage analysis described by Yan and colleagues. This technique has been labelled "mutated allele revealed by sequencing with aneuploidy and linkage analyses" (MARSALA) (Yan et al., 
What is Karyomapping and where does it fit in the world of preimplantation genetic diagnosis (PGD)?

2015) and involves multiple annealing and looping-based amplification cycles (MALBAC) for whole-genome amplification. Aneuploidy is then detected by copy number variations (CNVs), then detection of single-nucleotide variations (SNVs) in the PCR amplified MALBAC product determines the disease status of the sample. The false-positive and false-negative SNVs are avoided by using an NGS based linkage analysis (Yan et al., 2015), and importantly this technology has been used to achieve two viable and healthy live births (Yan et al., 2015).

Haplarithmisis (Zamani Esteki, 2015b; Zamani Esteki et al., 2015a) is an extension of Karyomapping technology that allows B allele frequencies to be called as well as the standard $\mathrm{AA}, \mathrm{BB}$ or $\mathrm{AB}$ alleles we expect from SNP data. Zamani et al. argue that the process of whole-genome amplification is in itself problematic due to the introduction of artefacts, thus other haplotyping methods suffer from error-prone SNP genotypes (AA, AB, BB) (Zamani Esteki, 2015b; Zamani Esteki et al., 2015a). Haplarithmisis has been shown to diagnose specific disease causing alleles throughout the genome, as well as indicating the presence of numerical and structural chromosomal abnormalities in the embryos. Furthermore, it has been shown that using this technique, meiotic segregation errors can be distinguished from mitotic ones (Zamani Esteki, 2015b).

\section{State of the ART}

Karyomapping was first commercialized by Illumina in 2013 and is currently a routine procedure for PGD detection of single gene disorders. At time of writing (April 2017) around 1000 clinics worldwide offer karyomapping, handled largely by 20 diagnostic laboratories (personal communication from PGD international society meeting, Valencia March 2017) with 2500 cycles currently performed. The inability to detect postzygotic trisomy reliably however and the ever-present problem of mosaicism means that Karyomapping is not widely used yet for aneuploidy screening. Presentations at the Valencia meeting are however describing strategies of combining NGS with Karyomapping so that copy number difference (meiotic and post-zygotic) can be detected alongside monogenic defects.

\section{Acknowledgments}

The authors are grateful to Professor Alan Handyside whose genius led to the invention of Karyomapping. We are also grateful to Cagri Ogur (Istanbul) for her critical reading of the manuscript and to Matt Wyatt (Genesis Genetics) for providing the Karyomapping screenshots. 
What is Karyomapping and where does it fit in the world of preimplantation genetic diagnosis (PGD)?

\section{References}

Ao, A., D. Wells, A.H. Handyside, R.M. Winston, and J.D. Delhanty. 1998. Preimplantation genetic diagnosis of inherited cancer: familial adenomatous polyposis coli. Journal of assisted reproduction and genetics. 15:140-144.

Ata, B., B. Kaplan, H. Danzer, M. Glassner, M. Opsahl, S.L. Tan, and S. Munné. 2012. Array CGH analysis shows that aneuploidy is not related to the number of embryos generated. Reproductive biomedicine online. 24:614-620.

Ben-Nagi, J., P. Serhal, S. SenGupta, K. Doye, and D. Wells. 2016. Preimplantation genetic diagnosis: an overview and recent advances. The Obstetrician \& Gynaecologist. 18:99-106.

Blockeel, C., V. Schutyser, A. De Vos, W. Verpoest, M. De Vos, C. Staessen, P. Haentjens, J. Van der Elst, and P. Devroey. 2008. Prospectively randomized controlled trial of PGS in IVF/ICSI patients with poor implantation. Reproductive biomedicine online. 17:848-854.

Braude, P., S. Pickering, F. Flinter, and C.M. Ogilvie. 2002. Preimplantation genetic diagnosis. Nature Reviews Genetics. 3:941-955.

Chong, S.S., K. Kristjansson, J. Cota, A.H. Handyslde, and M.R. Hughes. 1993. Preimplantation prevention of $\mathrm{X}$-linked disease: reliable and rapid sex determination of single human cells by restriction analysis of simultaneously amplified ZFX and ZFY sequences. Human molecular genetics. 2:1187-1191.
Dahdouh, E.M., J. Balayla, F. Audibert, R.D. Wilson, J.-A. Brock, C. Campagnolo, J. Carroll, K. Chong, A. Gagnon, and J.-A. Johnson. 2015. Technical update: preimplantation genetic diagnosis and screening. Obstetrical \& Gynecological Survey. 70:557-558.

De Ravel, T.J., K. Devriendt, J.-P. Fryns, and J.R. Vermeesch. 2007. What's new in karyotyping? The move towards array comparative genomic hybridisation (CGH). European journal of pediatrics. 166:637-643.

De Rycke, M. 2010. Singling out genetic disorders and disease. Genome medicine. 2:1.

Debrock, S., C. Melotte, C. Spiessens, K. Peeraer, E. Vanneste, L. Meeuwis, C. Meuleman, J.-P. Frijns, J.R. Vermeesch, and T.M. D'Hooghe. 2010. Preimplantation genetic screening for aneuploidy of embryos after in vitro fertilization in women aged at least 35 years: a prospective randomized trial. Fertility and sterility. 93:364-373.

Delhanty, J.D.A., D.K. Griffin, A.H. Handyside, J. Harper, G.H.G. Atkinson, M.H.E.C. Pieters, and R.M.L. Winston. 1993. Detection of aneuploidy and chromosomal mosaicism in human embryos during preimplantation sex determination by fluorescent in situ hybridisation,(FISH). Human molecular genetics. 2:1183.

Fiorentino, F., A. Biricik, S. Bono, L. Spizzichino, E. Cotroneo, G. Cottone, F. Kokocinski, and C.-E. Michel. 2014. Development and 
What is Karyomapping and where does it fit in the world of preimplantation genetic diagnosis (PGD)?

validation of a next-generation sequencing-based protocol for 24chromosome aneuploidy screening of embryos. Fertility and sterility. 101:1375-1382. e1372.

Fiorentino, F., A. Biricik, H. Karadayi, H. Berkil, G. Karlikaya, S. Sertyel, D. Podini, M. Baldi, M. Magli, and L. Gianaroli. 2004. Development and clinical application of a strategy for preimplantation genetic diagnosis of single gene disorders combined with HLA matching. Molecular Human Reproduction.

Fishel, S., A. Gordon, C. Lynch, K. Dowell, G. Ndukwe, E. Kelada, S. Thornton, L. Jenner, E. Cater, and A. Brown. 2010. Live birth after polar body array comparative genomic hybridization prediction of embryo ploidy - the future of IVF? Fertility and sterility. 93:1006. e1007-1006. e1010.

Forman, E.J., K.H. Hong, K.M. Ferry, X. Tao, D. Taylor, B. Levy, N.R. Treff, and R.T. Scott. 2013a. In vitro fertilization with single euploid blastocyst transfer: a randomized controlled trial. Fertility and sterility. 100:100-107. e101.

Forman, E.J., K.M. Upham, M. Cheng, T. Zhao, K.H. Hong, N.R. Treff, and R.T. Scott. 2013b. Comprehensive chromosome screening alters traditional morphology-based embryo selection: a prospective study of 100 consecutive cycles of planned fresh euploid blastocyst transfer. Fertility and sterility. 100:718-724.

Forozan, F., R. Karhu, J. Kononen, A. Kallioniemi, and O.-P. Kallioniemi. 1997. Genome screening by comparative genomic hybridization. Trends in Genetics. 13:405-409.

Frumkin, T., M. Malcov, Y. Yaron, and D. Ben-Yosef. 2008. Elucidating the origin of chromosomal aberrations in IVF embryos by preimplantation genetic analysis. Molecular and cellular endocrinology. 282:112-119.

Gardner, R., and R. Edwards. 1968. Rabbit by transferring Sexed Blastocysts. Nature. 218.

Geraedts, J., and G. De Wert. 2009. Preimplantation genetic diagnosis. Clinical genetics. 76:315-325.

Geraedts, J.P. 2010. Does additional hybridization also improve preimplantation genetic screening results?

Giménez, C., J. Sarasa, C. Arjona, E. Vilamajó, O. Martínez-Pasarell, K. Wheeler, G. Valls, E. GarciaGuixé, and D. Wells. 2015. Karyomapping allows preimplantation genetic diagnosis of a de-novo deletion undetectable using conventional PGD technology. Reproductive biomedicine online. 31:770-775.

Griffin, D., A. Handyside, R. Penketh, R. Winston, and J. Delhanty. 1991. Fluorescent in-situ hybridization to interphase nuclei of human preimplantation embryos with $\mathrm{X}$ and Y chromosome specific probes. Human Reproduction. 6:101-105.

Griffin, D.K., A.H. Handyside, J.C. Harper, L.J. Wilton, G. Atkinson, I. Soussis, D. Wells, E. Kontogianni, J. Tarin, and S. Geber. 1994. Clinical experience with preimplantation diagnosis of sex by dual fluorescent in situ Page | 11 
What is Karyomapping and where does it fit in the world of preimplantation genetic diagnosis (PGD)?

hybridization. Journal of assisted reproduction and genetics. 11:132143.

Griffin, D.K., L.J. Wilton, A.H. Handvside, R.M. Winston, and J.D. Delhanty. 1992. Dual fluorescent in situ hybridisation for simultaneous detection of $\mathrm{X}$ and $\mathrm{Y}$ chromosome-specific probes for the sexing of human preimplantation embryonic nuclei. Human genetics. 89:18-22.

\section{Griffin, D.K., L.J. Wilton, A.H.} Handyside, G. Atkinson, R. Winston, and J. Delhanty. 1993. Diagnosis of sex in preimplantation embryos by fluorescent in situ hybridisation. Bmj. 306:1382-1382.

Habela, C.W., and A. Hamosh. 2013. Genetic testing for intellectual disability: A role in diagnostic evaluation.

Handyside, A.H. 2011. PGD and aneuploidy screening for 24 chromosomes by genome-wide SNP analysis: seeing the wood and the trees. Reproductive biomedicine online. 23:686-691.

Handyside, A.H. 2013. 24-chromosome copy number analysis: a comparison of available technologies. Fertility and sterility. 100:595-602.

Handyside, A.H. 2015. Live births following karyomapping-a "key" milestone in the development of preimplantation genetic diagnosis. Reproductive biomedicine online. 31:307-308.

Handyside, A.H., G.L. Harton, B. Mariani, A.R. Thornhill, N. Affara, M.-A. Shaw, and D.K. Griffin. 2010. Karyomapping: a universal method for genome wide analysis of genetic disease based on mapping crossovers between parental haplotypes. Journal of medical genetics:jmg. 2009.069971.

Handyside, A.H., E.H. Kontogianni, K. Hardy, and R. Winston. 1990. Pregnancies from biopsied human preimplantation embryos sexed by $\mathrm{Y}$ specific DNA amplification. Nature. 344:768-770.

Handyside, A.H., J.G. Lesko, J.J. Tarín, R.M. Winston, and M.R. Hughes. 1992. Birth of a normal girl after in vitro fertilization and preimplantation diagnostic testing for cystic fibrosis. New England Journal of Medicine. 327:905-909.

Handyside, A.H., M.D. Robinson, R.J. Simpson, M.B. Omar, M.-A. Shaw, J.G. Grudzinskas, and A. Rutherford. 2004. Isothermal whole genome amplification from single and small numbers of cells: a new era for preimplantation genetic diagnosis of inherited disease. Molecular human reproduction. 10:767-772.

Handyside, A.H., and D. Wells. 2013. Single nucleotide polymorphisms and next generation sequencing. In Human Gametes and Preimplantation Embryos. Springer. 135-145.

Handyside, A.H., and K. Xu. 2012. Preimplantation genetic diagnosis comes of age. In Seminars in reproductive medicine. Vol. 30. Thieme Medical Publishers. 255-258.

Hardarson, T., C. Hanson, K. Lundin, T. Hillensjö, L. Nilsson, J. Stevic, E. Reismer, K. Borg, M. Wikland, and C. Bergh. 2008. Preimplantation genetic screening in women of 
What is Karyomapping and where does it fit in the world of preimplantation genetic diagnosis (PGD)?

advanced maternal age caused a decrease in clinical pregnancy rate: a randomized controlled trial. Human reproduction. 23:2806-2812.

Harper, J., L. Wilton, J. TraegerSynodinos, V. Goossens, C. Moutou, S. SenGupta, T.P. Budak, P. Renwick, M. De Rycke, and J. Geraedts. 2012a. The ESHRE PGD Consortium: 10 years of data collection. Human reproduction update. 18:234-247.

Harper, J.C., and S.B. SenGupta. 2012b. Preimplantation genetic diagnosis: state of the art 2011. Human genetics. 131:175-186.

Harton, G., M. De Rycke, F. Fiorentino, C. Moutou, S. SenGupta, J. Traeger-Synodinos, and J. Harper. 2011a. ESHRE PGD consortium best practice guidelines for amplificationbased PGD. Human reproduction. 26:33-40.

Harton, G., M. Magli, K. Lundin, M. Montag, J. Lemmen, and J. Harper. 2011b. ESHRE PGD Consortium/Embryology Special Interest Group-best practice guidelines for polar body and embryo biopsy for preimplantation genetic diagnosis/screening (PGD/PGS). Human reproduction. 26:41-46.

Hashiba, T., K. Sueoka, M. Kuroshima, H. Asada, N. Kuji, and Y. Yoshimura. 1999. Accurate multiplex polymerase chain reaction assay for gender determination from a single cell. Gynecologic and obstetric investigation. 49:217-220.

Hellani, A., K. Abu-Amero, J. Azouri, and S. El-Akoum. 2008. Successful pregnancies after application of array-comparative genomic hybridization in PGS-aneuploidy screening. Reproductive biomedicine online. 17:841-847.

Hussey, N.D., H. Donggui, D.A. Froiland, D.J. Hussey, E.A. Haan, C.D. Matthews, and J.E. Craig. 1999. Analysis of five Duchenne muscular dystrophy exons and gender determination using conventional duplex polymerase chain reaction on single cells. Molecular human reproduction. 5:1089-1094.

International Human Genome Sequencing Consortium. 2004. Finishing the euchromatic sequence of the human genome. In Nature. Vol. 431. 931945.

Ioannou, D., K. Fonseka, E.J. Meershoek, A.R. Thornhill, A. Abogrein, M. Ellis, and D.K. Griffin. 2012. Twenty-four chromosome FISH in human IVF embryos reveals patterns of post-zygotic chromosome segregation and nuclear organisation. Chromosome research. 20:447-460.

Ioannou, D., E.J. Meershoek, A.R. Thornhill, M. Ellis, and D.K. Griffin. 2011. Multicolour interphase cytogenetics: 24 chromosome probes, 6 colours, 4 layers. Molecular and cellular probes. 25:199-205.

Jansen, R.P., M.C. Bowman, K.A. de Boer, D.A. Leigh, D.B. Lieberman, and S.J. McArthur. 2008. What next for preimplantation genetic screening (PGS)? Experience with blastocyst biopsy and testing for aneuploidy. Human reproduction. 23:1476-1478. 
What is Karyomapping and where does it fit in the world of preimplantation genetic diagnosis (PGD)?

Kahraman, S., C. Beyazyurek, M.A. Yesilipek, G. Ozturk, M. Ertem, S. Anak, S. Kansoy, S. Aksoylar, B. Kuşkonmaz, and H. Oniz. 2014. Successful haematopoietic stem cell transplantation in 44 children from healthy siblings conceived after preimplantation HLA matching. Reproductive biomedicine online. 29:340-351.

Kirchhoff, M., T. Gerdes, H. Rose, J. Maahr, A.M. Ottesen, and C. Lundsteen. 1998. Detection of chromosomal gains and losses in comparative genomic hybridization analysis based on standard reference intervals. Cytometry Part A. 31:163173.

Konstantinidis, M., R. Prates, N.-N. Goodall, J. Fischer, V. Tecson, T. Lemma, B. Chu, A. Jordan, E. Armenti, and D. Wells. 2015. Live births following Karyomapping of human blastocysts: experience from clinical application of the method. Reproductive biomedicine online. 31:394-403.

LaFramboise, T. 2009. Single nucleotide polymorphism arrays: a decade of biological, computational and technological advances. Nucleic acids research:gkp552.

Lander, E.S., L.M. Linton, B. Birren, C. Nusbaum, M.C. Zody, J. Baldwin, K. Devon, K. Dewar, M. Doyle, and W. FitzHugh. 2001. Initial sequencing and analysis of the human genome. Nature. 409:860921.

Le Caignec, C., C. Spits, K. Sermon, M. De Rycke, B. Thienpont, S. Debrock, C. Staessen, Y. Moreau, J.-P. Fryns, and A. Van
Steirteghem. 2006. Single-cell chromosomal imbalances detection by array CGH. Nucleic acids research. 34:e68-e68.

Levinson, G., K. Keyvanfar, J.C. Wu, E.F. Fugger, R.A. Fields, G.L. Harton, F.T. Palmer, M.E. Sisson, K.M. Starr, and L. Dennison-Lagos. 1995. Genetics and human conception: DNA-based X-enriched sperm separation as an adjunct to preimplantation genetic testing for the prevention of $\mathrm{X}$-linked disease. Human Reproduction. 10:979-982.

Lichter, P., S. Joos, M. Bentz, and S. Lampel. 2000. Comparative genomic hybridization: uses and limitations. In Seminars in hematology. Vol. 37. Elsevier. 348357.

Liu, J., W. Lissens, C. Van Broeckhoven, A. Löfgren, M. Camus, I. Liebaers, and A. Van Steirteghem. 1995. Normal pregnancy after preimplantation DNA diagnosis of a dystrophin gene deletion. Prenatal diagnosis. 15:351-358.

Mastenbroek, S., M. Twisk, J. van Echten-Arends, B. SikkemaRaddatz, J.C. Korevaar, H.R. Verhoeve, N.E. Vogel, E.G. Arts, J.W. De Vries, and P.M. Bossuyt. 2007. In vitro fertilization with preimplantation genetic screening. New England Journal of Medicine. 357:9-17.

Mersereau, J.E., E. Pergament, X. Zhang, and M.P. Milad. 2008. Preimplantation genetic screening to improve in vitro fertilization pregnancy rates: a prospective randomized controlled trial. Fertility and sterility. 90:1287-1289. 
What is Karyomapping and where does it fit in the world of preimplantation genetic diagnosis (PGD)?

Meyer, L.R., S. Klipstein, W.D. Hazlett, T. Nasta, P. Mangan, and V.C. Karande. 2009. A prospective randomized controlled trial of preimplantation genetic screening in the "good prognosis" patient. Fertility and sterility. 91:1731-1738.

Munné, S. 2006. Chromosome abnormalities and their relationship to morphology and development of human embryos. Reproductive biomedicine online. 12:234-253.

Munné, S., M. Alikani, G. Tomkin, J. Grifo, and J. Cohen. 1996. Embryo morphology, developmental rates, and maternal age are correlated with chromosome abnormalities. International Journal of Gynecology and Obstetrics. 52:329-329.

Munné, S., and J. Cohen. 1993. Unsuitability of multinucleated human blastomeres for preimplantation genetic diagnosis. Human Reproduction. 8:1120-1125.

Munné, S., and J. Cohen. 1998. Chromosome abnormalities in human embryos. Human Reproduction Update. 4:842-855.

Munné, S., H. Weier, J. Grifo, and J. Cohen. 1994. Chromosome mosaicism in human embryos. Biology of Reproduction. 51:373379.

Nakahori, Y., K. Hamano, M. Iwaya, and Y. Nakagome. 1991. Sex identification by polymerase chain reaction using $\mathrm{X}-\mathrm{Y}$ homologous primer. American journal of medical genetics. 39:472-473.

Natesan, S.A., A.J. Bladon, S. Coskun, W. Qubbaj, R. Prates, S. Munne, E. Coonen, J.C. Dreesen, S.J. Stevens, and A.D. Paulussen. 2014a. Genome-wide karyomapping accurately identifies the inheritance of single-gene defects in human preimplantation embryos in vitro. Genetics in Medicine.

Natesan, S.A., A.H. Handyside, A.R. Thornhill, C.S. Ottolini, K. Sage, M.C. Summers, M. Konstantinidis, D. Wells, and D.K. Griffin. 2014b. Live birth after PGD with confirmation by a comprehensive approach (karyomapping) for simultaneous detection of monogenic and chromosomal disorders. Reproductive biomedicine online. 29:600-605.

Rabinowitz, M., D. Potter, N. Wemmer, Z. Demko, and G. Gemelos. 2011. First clinical outcomes reported on patients undergoing PGD for genetic disorders together with 24 chromosome ploidy using microarrays. Fertility and Sterility. 95:S6-S7.

Rabinowitz, M., A. Ryan, G. Gemelos, M. Hill, J. Baner, C. Cinnioglu, M. Banjevic, D. Potter, D.A. Petrov, and Z. Demko. 2012. Origins and rates of aneuploidy in human blastomeres. Fertility and sterility. 97:395-401.

Ray, P.F., M. Vekemans, and A. Munnich. 2001. Single cell multiplex PCR amplification of five dystrophin gene exons combined with gender determination. Molecular human reproduction. 7:489-494.

Rechitsky, S., T. Pakhalchuk, G. San Ramos, A. Goodman, Z. Zlatopolsky, and A. Kuliev. 2015. First systematic experience of preimplantation genetic diagnosis for 
What is Karyomapping and where does it fit in the world of preimplantation genetic diagnosis (PGD)?

single-gene disorders, and/or preimplantation human leukocyte antigen typing, combined with 24chromosome aneuploidy testing. Fertility and sterility. 103:503-512.

Rechitsky, S., E. Pomerantseva, T. Pakhalchuk, D. Pauling, O. Verlinsky, and A. Kuliev. 2011. First systematic experience of preimplantation genetic diagnosis for de-novo mutations. Reproductive biomedicine online. 22:350-361.

Renwick, P., J. Trussler, A. Lashwood, P. Braude, and C.M. Ogilvie. 2010. Preimplantation genetic haplotyping: 127 diagnostic cycles demonstrating a robust, efficient alternative to direct mutation testing on single cells. Reproductive biomedicine online. 20:470-476.

Sachidanandam, R., D. Weissman, S.C. Schmidt, J.M. Kakol, L.D. Stein, G. Marth, S. Sherry, J.C. Mullikin, B.J. Mortimore, and D.L. Willey. 2001. A map of human genome sequence variation containing 1.42 million single nucleotide polymorphisms. Nature. 409:928933.

Schoolcraft, W.B., M.G. Katz-Jaffe, J. Stevens, M. Rawlins, and S. Munne. 2009. Preimplantation aneuploidy testing for infertile patients of advanced maternal age: a randomized prospective trial. Fertility and sterility. 92:157-162.

Scott, R.T., K.M. Upham, E.J. Forman, K.H. Hong, K.L. Scott, D. Taylor, X. Tao, and N.R. Treff. 2013b. Blastocyst biopsy with comprehensive chromosome screening and fresh embryo transfer significantly increases in vitro fertilization implantation and delivery rates: a randomized controlled trial. Fertility and sterility. 100:697-703.

Spelcher, M.R., S. Du Manoir, E. SchrÖck, H. Holtgreve-Grez, B. Schoell, C. Lengauer, T. Cremer, and T. Ried. 1993. Molecular cytogenetic analysis of formalinfixed, paraffin-embedded solid tumors by comparative genomic hybridization after universal DNAamplification. Human Molecular Genetics. 2:1907-1914.

Spits, C., and K. Sermon. 2009. PGD for monogenic disorders: aspects of molecular biology. Prenatal diagnosis. 29:50-56.

Staessen, C., W. Verpoest, P. Donoso, P. Haentjens, J. Van der Elst, I. Liebaers, and P. Devroey. 2008. Preimplantation genetic screening does not improve delivery rate in women under the age of 36 following single-embryo transfer. Human Reproduction. 23:2818-2825.

Summers, M.C., and A.D. Foland. 2009. Quantitative decision-making in preimplantation genetic (aneuploidy) screening (PGS). Journal of assisted reproduction and genetics. 26:487502.

Theisen, A. 2008. Microarray-based comparative genomic hybridization (aCGH). Nature Education. 1:45.

Thornhill, A.R., A.H. Handyside, C. Ottolini, S.A. Natesan, J. Taylor, K. Sage, G. Harton, K. Cliffe, N. Affara, and M. Konstantinidis. $2015 . \quad$ Karyomapping-a comprehensive means of simultaneous monogenic and 
What is Karyomapping and where does it fit in the world of preimplantation genetic diagnosis (PGD)?

cytogenetic PGD: comparison with standard approaches in real time for Marfan syndrome. Journal of assisted reproduction and genetics. 32:347-356.

Thornhill, A.R., and K. Snow. 2002. Molecular diagnostics in preimplantation genetic diagnosis. The Journal of molecular diagnostics. 4:11-29.

Traeger-Synodinos, J., and C. Staessen. 2014. Preimplantation genetic diagnosis. Textbook of Human Reproductive Genetics: 157.

Traversa, M.V., J. Marshall, S. McArthur, and D. Leigh. 2011. The genetic screening of preimplantation embryos by comparative genomic hybridisation. Reprod Biol. 11:51-60.

Treff, N.R., A. Fedick, X. Tao, B. Devkota, D. Taylor, and R.T. Scott. 2013a. Evaluation of targeted nextgeneration sequencing-based preimplantation genetic diagnosis of monogenic disease. Fertility and sterility. 99:1377-1384. e1376.

Treff, N.R., L.E. Northrop, K. Kasabwala, J. Su, B. Levy, and R.T. Scott. 2011. Single nucleotide polymorphism microarray-based concurrent screening of 24chromosome aneuploidy and unbalanced translocations in preimplantation human embryos. Fertility and sterility. 95:1606-1612. e1602.

Treff, N.R., and R.T. Scott. 2013b. Fourhour quantitative real-time polymerase chain reaction-based comprehensive chromosome screening and accumulating evidence of accuracy, safety, predictive value, and clinical efficacy. Fertility and sterility. 99:1049-1053.

Treff, N.R., X. Tao, K.M. Ferry, J. Su, D. Taylor, and R.T. Scott. 2012. Development and validation of an accurate quantitative real-time polymerase chain reaction-based assay for human blastocyst comprehensive chromosomal aneuploidy screening. Fertility and sterility. 97:819-824. e812.

Vanneste, E., T. Voet, C. Le Caignec, M. Ampe, P. Konings, C. Melotte, S. Debrock, M. Amyere, M. Vikkula, and F. Schuit. 2009. Chromosome instability is common in human cleavage-stage embryos. Nature medicine. 15:577-583.

Venter, J.C., M.D. Adams, E.W. Myers, P.W. Li, R.J. Mural, G.G. Sutton, H.O. Smith, M. Yandell, C.A. Evans, and R.A. Holt. 2001. The sequence of the human genome. science. 291:1304-1351.

Yan, L., L. Huang, L. Xu, J. Huang, F. Ma, X. Zhu, Y. Tang, M. Liu, Y. Lian, and P. Liu. 2015. Live births after simultaneous avoidance of monogenic diseases and chromosome abnormality by next-generation sequencing with linkage analyses. Proceedings of the National Academy of Sciences. 112:1596415969.

Yang, Z., J. Liu, G.S. Collins, S.A. Salem, X. Liu, S.S. Lyle, A.C. Peck, E.S. Sills, and R.D. Salem. 2012. Selection of single blastocysts for fresh transfer via standard morphology assessment alone and with array CGH for good prognosis IVF patients: results from a 
What is Karyomapping and where does it fit in the world of preimplantation genetic diagnosis (PGD)?

randomized pilot study. Molecular cytogenetics. 5:1-8.

Zamani Esteki, M. 2015b. Haplarithmisis to study haplotypes genome-wide to single-cell resolution, enabling a generic method for preimplantation genectic diagnosis in the clinic and novel fundamental genome research.

Zamani Esteki, M., E. Dimitriadou, L. Mateiu, C. Melotte, N. Van der Aa, P. Kumar, R. Das, K. Theunis, J. Cheng, and E. Legius. 2015a. Concurrent whole-genome haplotyping and copy-number profiling of single cells. The
American Journal of Human Genetics. 96:894-912.

Zimmerman, R.S., C. Jalas, X. Tao, A.M. Fedick, J.G. Kim, R.J. Pepe, L.E. Northrop, R.T. Scott, and N.R. Treff. 2016. Development and validation of concurrent preimplantation genetic diagnosis for single gene disorders and comprehensive chromosomal aneuploidy screening without whole genome amplification. Fertility and sterility.

105:286-294. 